Supporting Information

\title{
PEP27-2, a potent antimicrobial cell-penetrating peptide, reduces skin abscess formation during Staphylococcus aureus infections in mouse, when used in combination with antibiotics
}

Hee Kyoung Kang ${ }^{1}$, Jonggwan Park ${ }^{2}$, Chang Ho Seo ${ }^{2}$ and Yoonkyung Park ${ }^{1,3, *}$

${ }^{1}$ Department of Biomedical Science, Chosun University, Gwangju 61452, Korea

${ }^{2}$ Department of Bioinformatics, Kongju National University, Kongju 32588, Korea

${ }^{3}$ Research Center for Proteineous Materials (RCPM), Chosun University, Gwangju 61452,

Korea

*E-mail:y_k_park@chosun.ac.kr; Tel.: +82-62-230-6854; Fax: +82-62-225-6758

Table of contents:

Figure S1. Analytical RP-HPLC and mass spectra of PEP27, PEP27-1, PEP27-2 and PEP275 , respectively S3

Figure S2. Intercellular delivery of PEP27-2 into MCF-7 cells $\cdot$ S5 
Figure S3. Relative fluorescence intensity was calculated with Celleste ${ }^{\mathrm{TM}}$ Image Analysis Software S6

Figure S4. FIC isobolograms showing interactions between antibiotics and AMP during inhibition of S. aureus MW2 growth S7

Figure S5. PI uptake assay of synergistic effect of the PEP27-2 and ceftriaxone S8

Figure S6. Action mechanism of ceftriaxone .S9

Figure S7. Internalization of PEP27-2 and TAT in MRSA S10

Table S1. MBIC of PEP27-2 against various microorganisms S11

Table S2. MIC of PEP27-2 and antibiotics against S. aureus and P. aeruginosa S12

Table S3. Combination activity of PEP27-2 with meropenem, ceftazidime, and ceftriaxone against $S$. aureus S13

Table S4. Combination activity of PEP27-2 with meropemem, ceftazidime and ceftriaxone against $P$. aeruginosa $\cdot$ S14

Table S5. MICDMEM value and FIC for PEP27-2 and antibiotics against S. aureus MW2-GFP S15

Table S6. List of bacterial strains used in this study S16

Table S7. Primers used in qPCR to evaluate inflammatory cytokines S17

References. S18 
1. Analytical RP-HPLC and mass spectra of PEP27
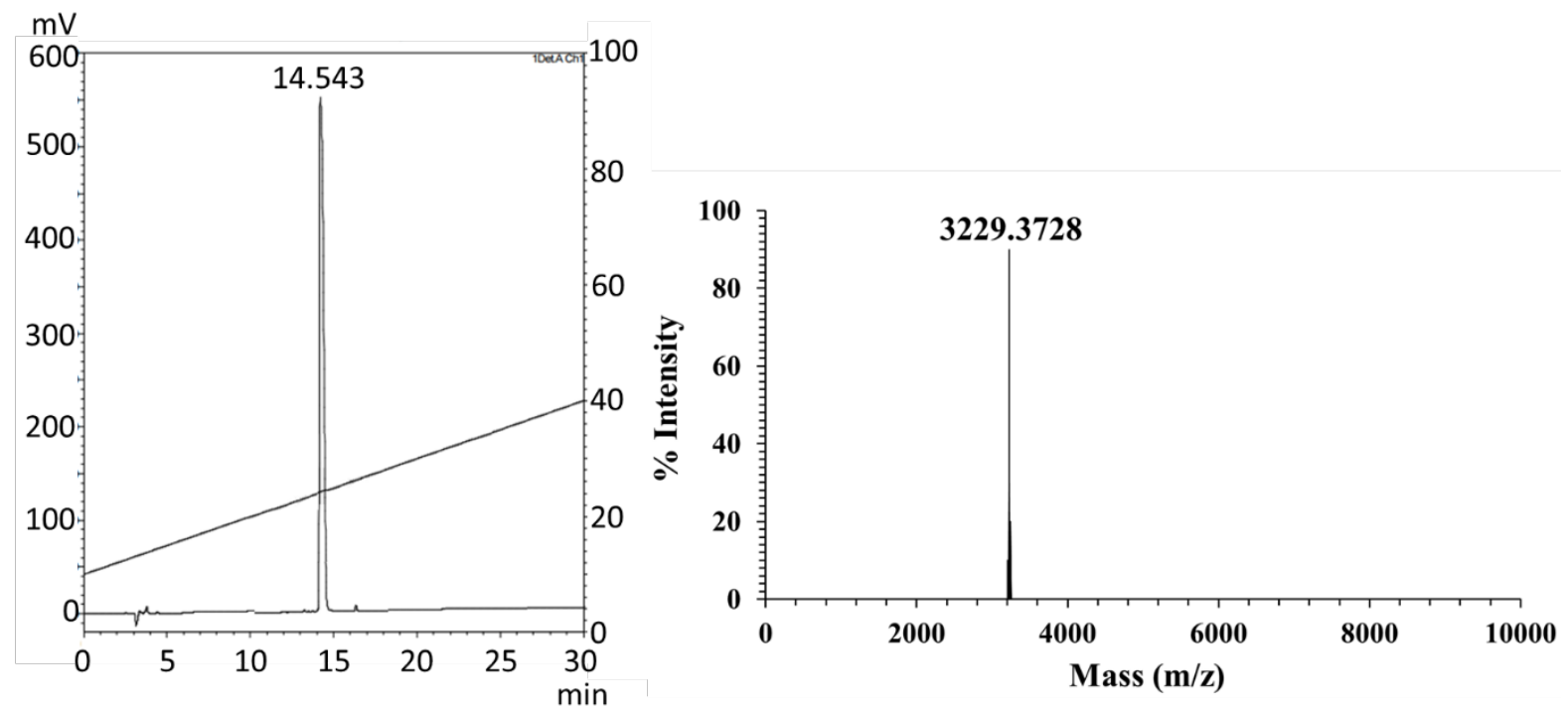

2. Analytical RP-HPLC and mass spectra of PEP27-1

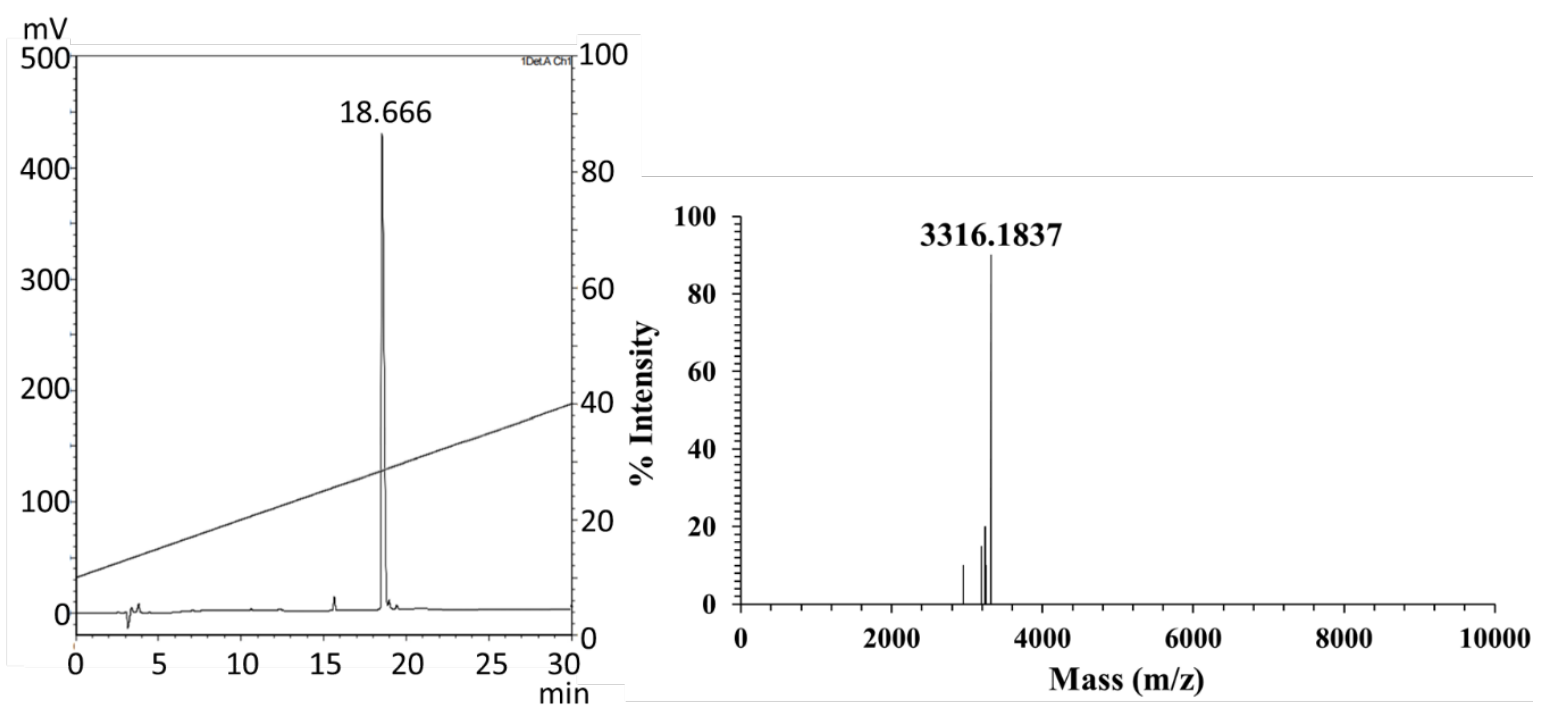


3. Analytical RP-HPLC and mass spectra of PEP27-2

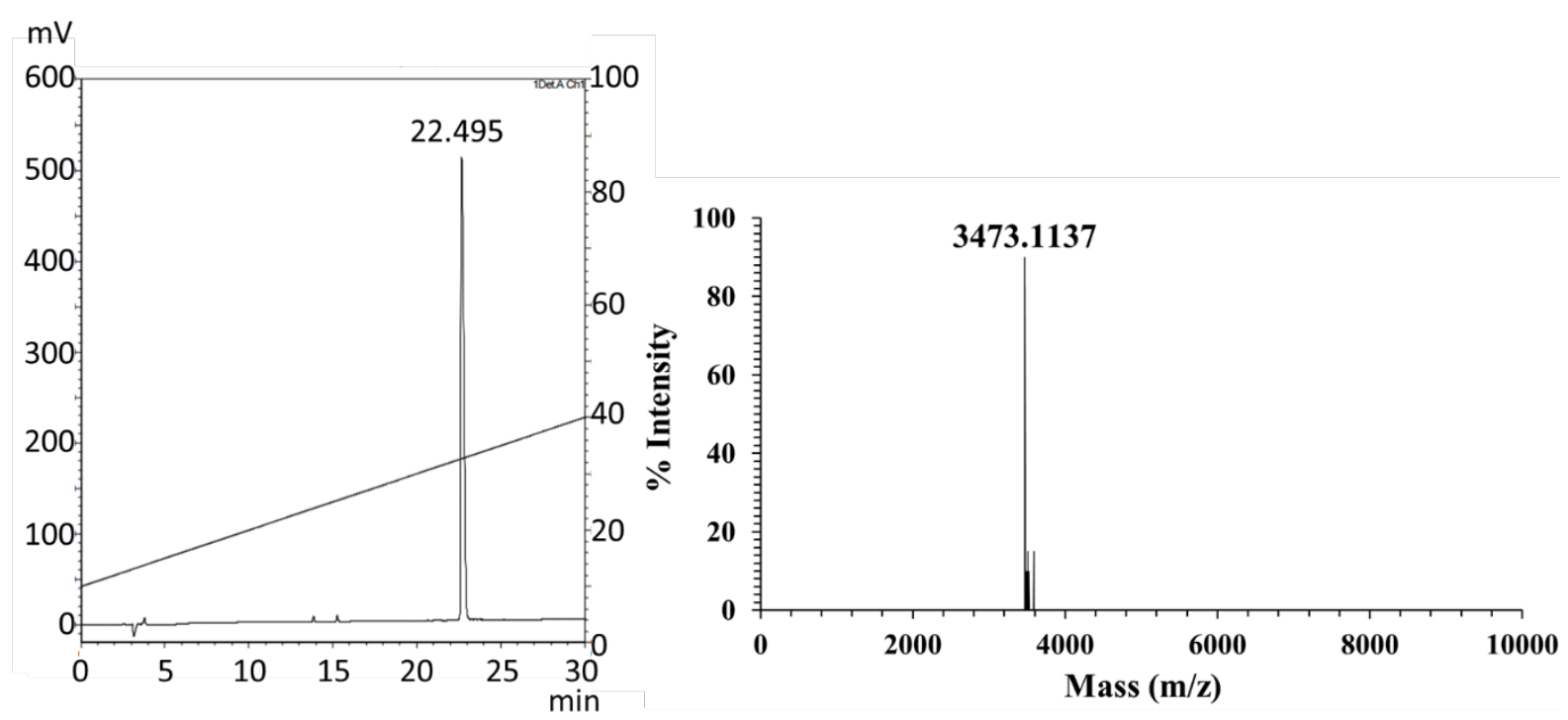

4. Analytical RP-HPLC and mass spectra of PEP27-5
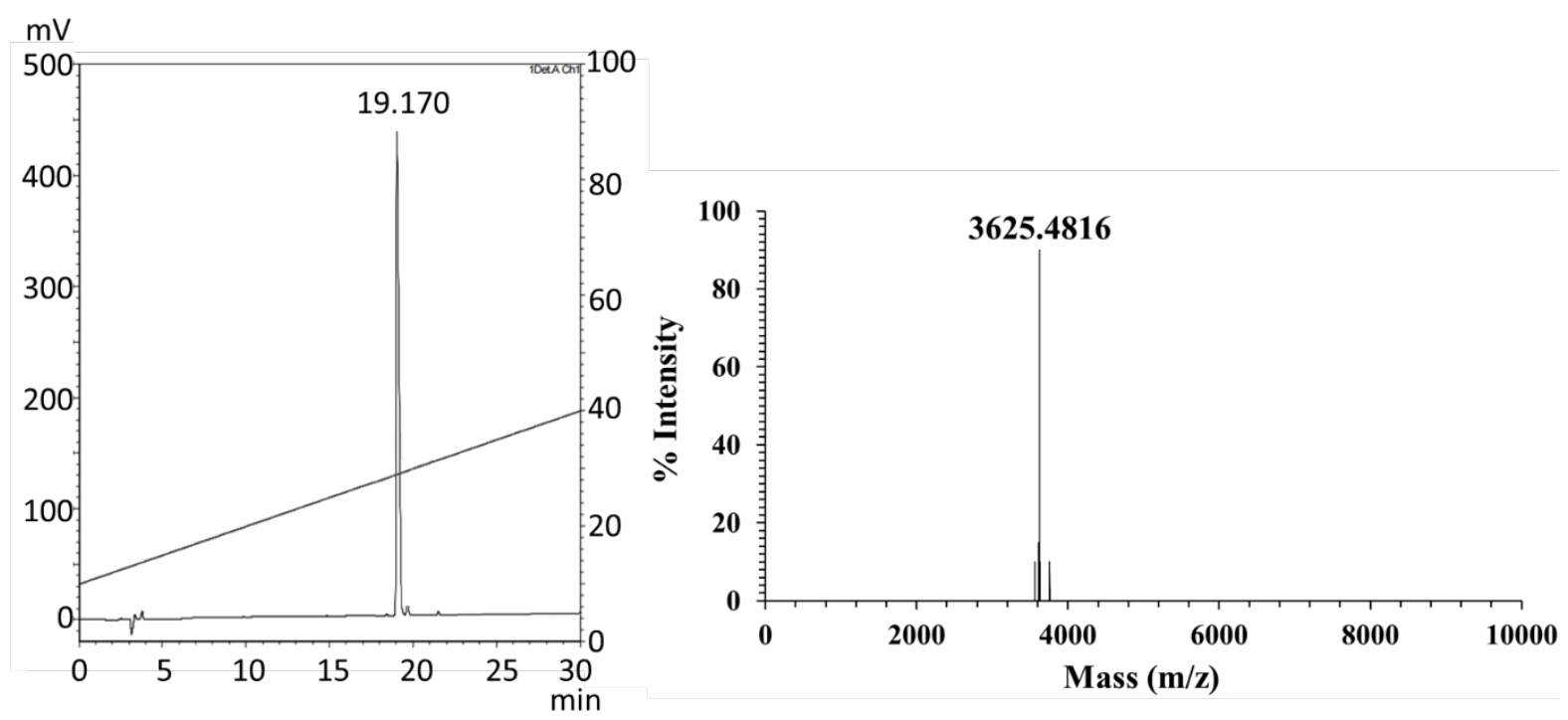

Figure S1. Analytical RP-HPLC and mass spectra of PEP27, PEP27-1, PEP27-2 and PEP275, respectively. The elution of the peptides was monitored at $215 \mathrm{~nm}$. 


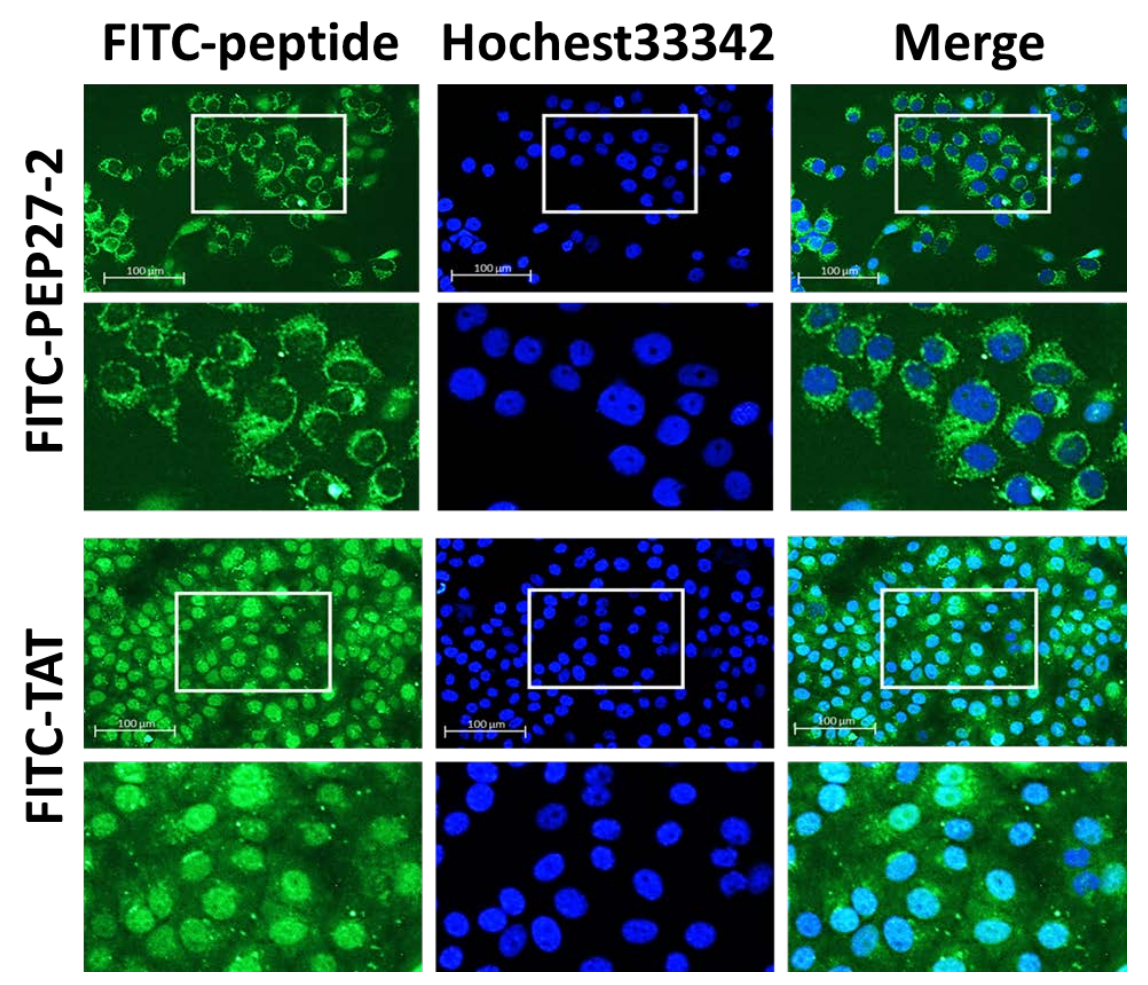

Figure S2. Intercellular delivery of PEP27-2 into MCF7 cells. Transduction of FITC-labeled PEP27-2 into MCF7 cells ( $\mathrm{n}=4$ images per condition). MCF7 cells were incubated with FITClabeled PEP27-2 or FITC-labeled TAT (green), followed by counterstaining with Hoechst 33342 (blue). Uptake was monitored by fluorescence using a phase-contrast microscope (EVOSTM FL Auto 2 Imaging System). The left figures show the FITC-labeled peptide internalized in the cells with prevalent submembrane localization. The middle figures show cell nuclei stained with Hoechst 33342 (blue). The right figures show the overlay between the two images. These experiments were repeated three times and showed similar results. 


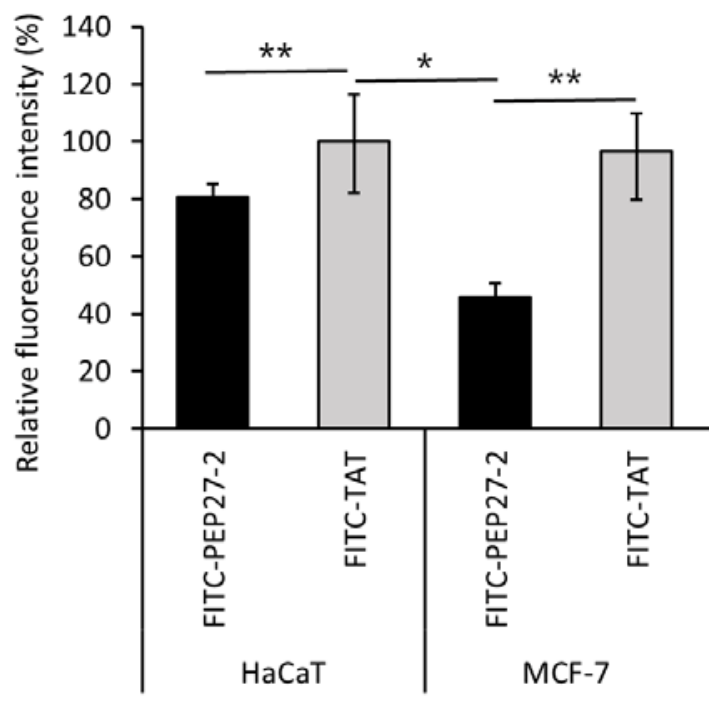

Figure S3. Relative fluorescence intensity was calculated with Celleste ${ }^{\mathrm{TM}}$ Image Analysis Software. Values shown are the mean $\pm \mathrm{SD}$ (HaCaT, $\mathrm{n}=3$ and $\mathrm{MCF}, \mathrm{n}=4$ image for condition). There was a significant difference between different FITC-labeled peptides in the same cell line as determined by one-way ANOVA $(* * P<0.01, * P<0.05)$. 

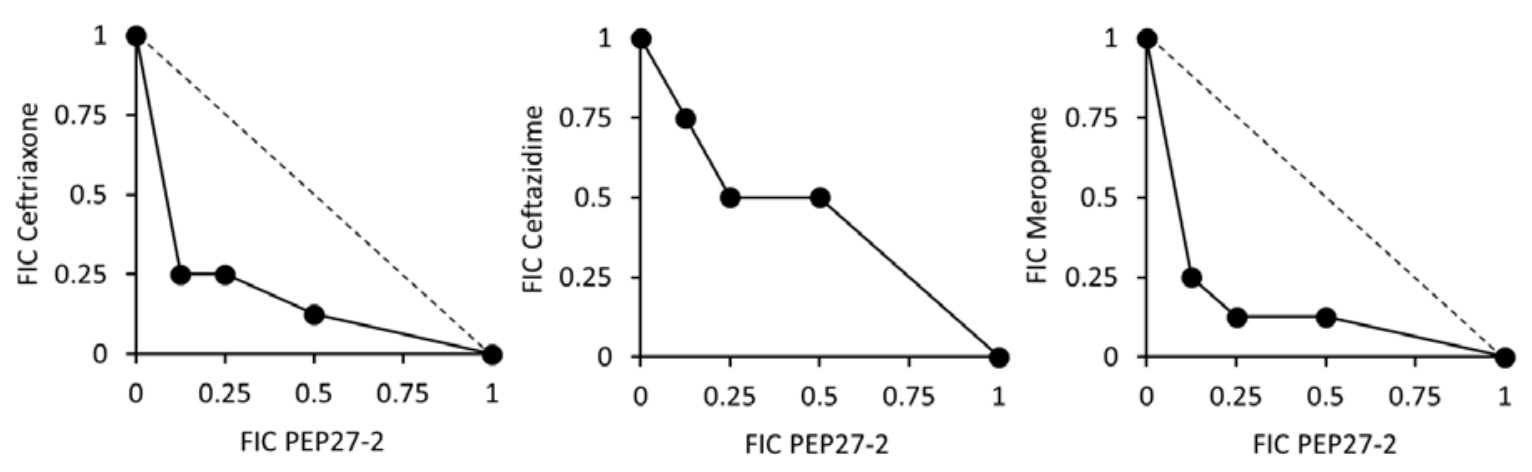

Figure S4. FIC isobolograms showing interactions between antibiotics and AMP during inhibition of S. aureus MW2 growth. FIC values derived from the combinations of ceftriaxone, ceftazidime, meropeme, and PEP27-2 were used to plot the isobolograms. An FIC value of 1 corresponds to the MIC values of the specific antibiotics or AMP. The dotted line represents the theoretical additive interaction between antibiotics and AMP. 


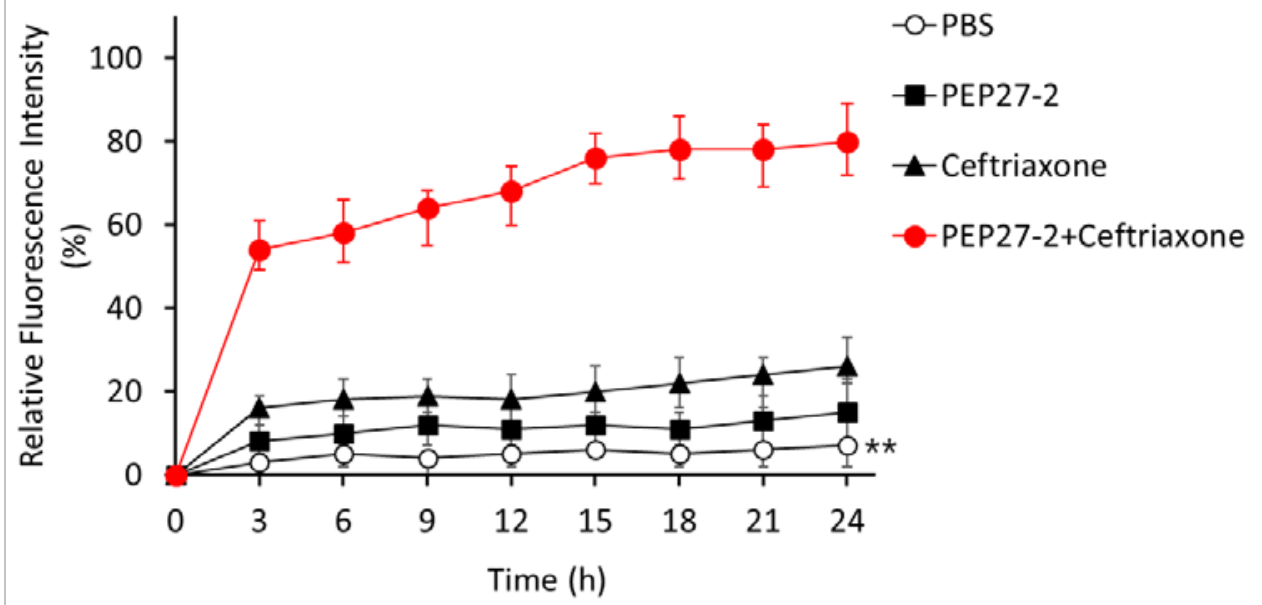

Figure S5. PI uptake assay of synergistic effect of PEP27-2 and ceftriaxone ( $n=3$ per condition). S. aureus MW2 treated with $0.25 \times$ PEP27-2 alone, $0.5 \times$ ceftriaxone alone as control, and both combine at same concentration taken as test. Data are presented as the means \pm SD. ${ }^{* *} P<0.01$ vs untreated control. Data were analyzed by one-way ANOVA. 


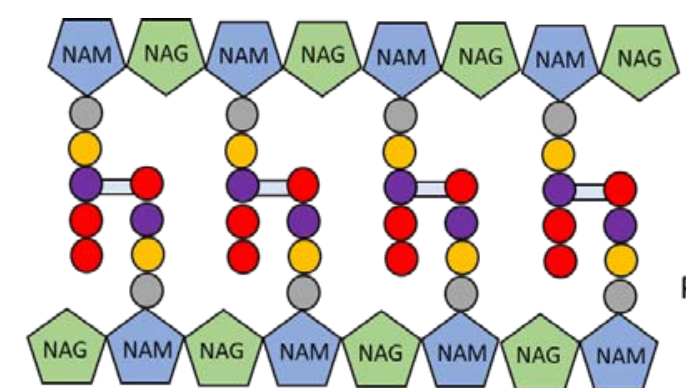

Cell wall synthesis

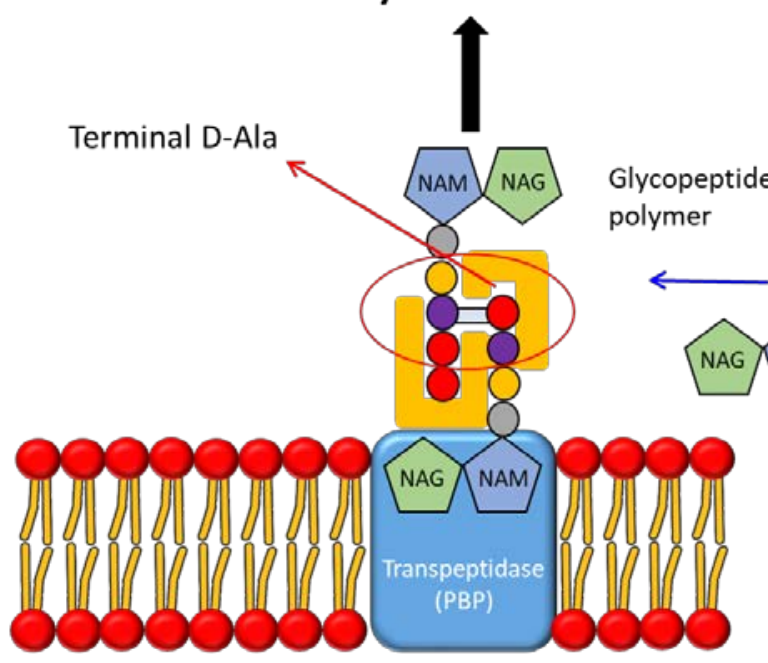

Peptidoglycan

layer
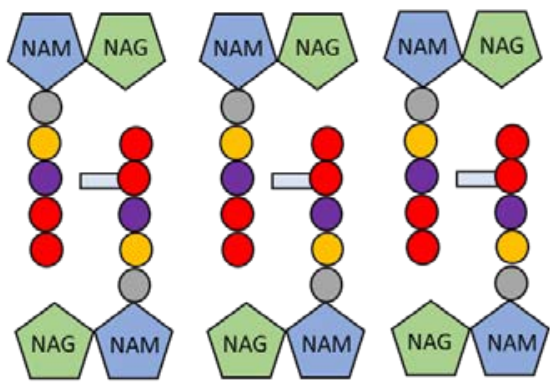

Block of PBP activity interrupts cross-linking and cell wall synthesis

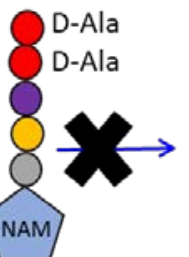

me inhibited

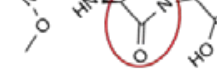

$\beta$-lactam : bind to PBP active site
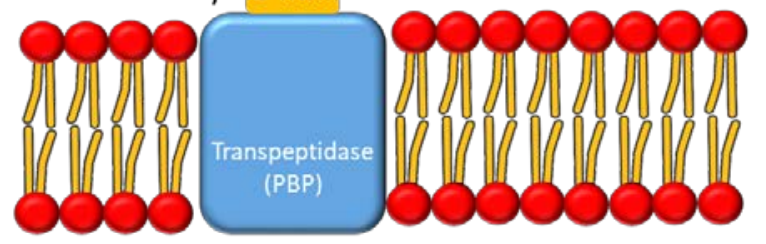

Bactericidal effect

Cytosol

Figure S6. Action mechanism of ceftriaxone. Ceftriaxone binds to and inactivates penicillinbinding protein (PBP) on the inner membrane of the bacterial cell wall. PBPs participate in the terminal stages of assembly of the bacterial cell wall and in reshaping of the cell wall during cell division. Inactivation of PBPs interferes with the cross-linkage of peptidoglycan chains necessary for bacterial cell wall strength and rigidity. This results in weakening of the bacterial cell wall and causes cell lysis. 


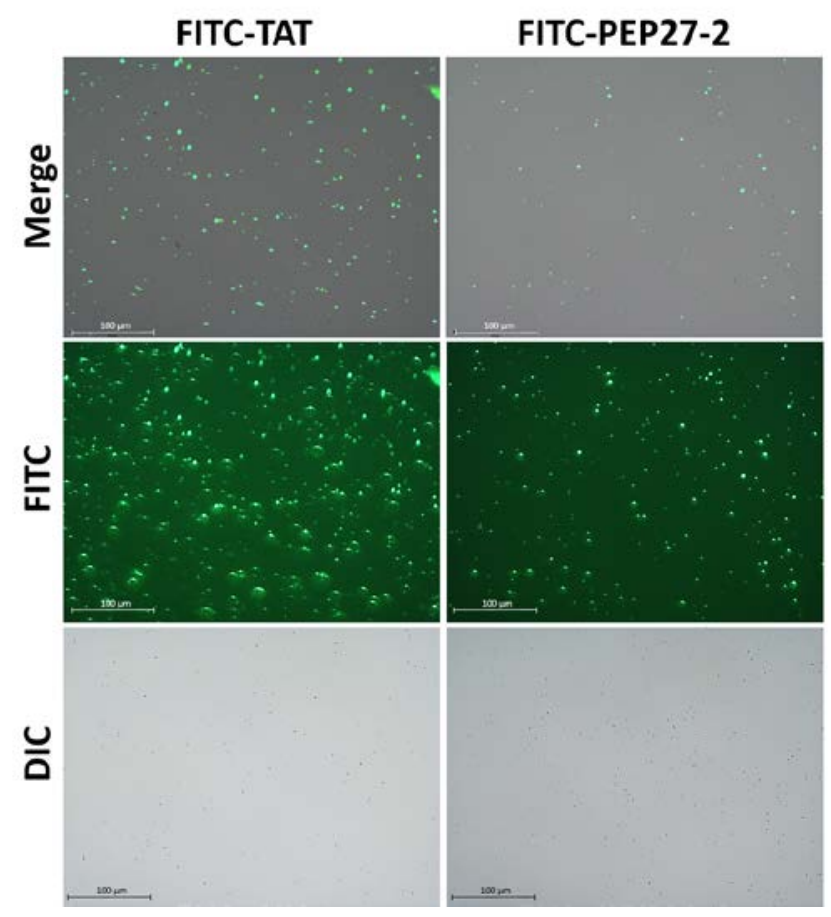

Figure S7. Internalization of PEP27-2 and TAT in MRSA ( $n=3$ per condition). Bacterial cells were incubated at $37^{\circ} \mathrm{C}$ for $1 \mathrm{~h}$ in PBS containing the FITC-labeled PEP27-2 and TAT (2 $\mu \mathrm{M}$ each). At the end of the incubation period, bacterial cells were washed twice with PBS, treated with trysin, and re-suspended in PBS. Finally, cells were monitored by fluorescence using a phase-contrast microscope (EVOS ${ }^{\mathrm{TM}}$ FL Auto 2 Imaging System, Thermo Fisher Scientific). 
Table S1. MBIC of PEP27-2 against various microorganisms

\begin{tabular}{|c|c|c|}
\hline \multirow[t]{2}{*}{ Microorganism } & \multicolumn{2}{|c|}{$\operatorname{MBIC}(\mu \mathrm{M})^{a}$} \\
\hline & PEP27-2 & Melittin \\
\hline \multicolumn{3}{|l|}{ Gram+ } \\
\hline S. aureus ATCC25923 & 4 & 4 \\
\hline S. aureus ATCC29213 & 4 & 4 \\
\hline \multicolumn{3}{|l|}{ Gram- } \\
\hline E. coli ATCC25922 & 8 & 4 \\
\hline E. coli ATCC27325 & 8 & 4 \\
\hline P. aeruginosa ATCC15692 & 8 & 4 \\
\hline P. aeruginosa ATCC27853 & 8 & 4 \\
\hline \multicolumn{3}{|l|}{ Resistant Gram+ } \\
\hline S. aureus CCARM3089 & 8 & 4 \\
\hline S. aureus CCARM3090 & 8 & 4 \\
\hline S. aureus CCARM3518 & 8 & 8 \\
\hline S. aureus USA300 & 4 & 4 \\
\hline S. aureus MW2 & 8 & 8 \\
\hline \multicolumn{3}{|l|}{ Resistant Gram- } \\
\hline E. coli CCARM1229 & 8 & 4 \\
\hline E. coli CCARM1238 & 8 & 4 \\
\hline P. aeruginosa 4007 & 8 & 4 \\
\hline P. aeruginosa 4891 & 8 & 8 \\
\hline
\end{tabular}

${ }^{a} \mathrm{MBIC}$ were determined in three independent experiments performed in triplicate. 
Table S2. MIC of PEP27-2 and antibiotics against S. aureus and P. aeruginosa

\begin{tabular}{|c|c|c|c|c|c|}
\hline \multirow[t]{2}{*}{ Microorganism } & \multicolumn{5}{|c|}{$\mathrm{MIC}(\mu \mathrm{M})$} \\
\hline & PEP27-2 & Meropenem & Ceftazidime & Ceftriaxone & Vancomycin \\
\hline S. aureus ATCC25923 & 2 & 2 & 16 & 8 & 0.25 \\
\hline S. aureus ATCC29213 & 2 & 2 & 8 & 4 & 0.25 \\
\hline S. aureus CCARM3089 & 4 & 64 & 512 & 512 & 0.25 \\
\hline S. aureus CCARM3090 & 4 & 128 & 512 & 512 & 0.5 \\
\hline S. aureus CCARM3518 & 4 & 64 & 64 & 256 & 0.25 \\
\hline S. aureus USA300 & 2 & 2 & 32 & 16 & 0.25 \\
\hline S. aureus MW2 & 4 & 2 & 32 & 32 & 0.25 \\
\hline P. aeruginosa ATCC15692 & 4 & 2 & 4 & 16 & 512 \\
\hline P. aeruginosa ATCC27853 & 4 & 2 & 2 & 8 & 256 \\
\hline P. aeruginosa 4007 & 4 & 4 & 16 & 16 & 512 \\
\hline P. aeruginosa 4891 & 4 & 8 & 16 & 64 & 512 \\
\hline
\end{tabular}


Table S3. Combination activity of PEP27-2 with meropenem, ceftazidime, and ceftriaxone against $S$. aureus

\begin{tabular}{|c|c|c|c|}
\hline Organism & Agent & FIC $^{a}$ & Combination activity \\
\hline \multirow[t]{3}{*}{ S. aureus ATCC25923 } & PEP27-2 / Meropemem & 1 & Indifferent \\
\hline & PEP27-2 / Ceftazidime & 0.75 & Indifferent \\
\hline & PEP27-2 / Ceftriaxone & 0.75 & Indifferent \\
\hline \multirow[t]{3}{*}{ S. aureus ATCC29213 } & PEP27-2 / Meropemem & 1 & Indifferent \\
\hline & PEP27-2 / Ceftazidime & 0.75 & Indifferent \\
\hline & PEP27-2 / Ceftriaxone & 0.5 & Synergy \\
\hline \multirow[t]{3}{*}{ S. aureus USA300 } & PEP27-2 / Meropemem & 0.75 & Indifferent \\
\hline & PEP27-2 / Ceftazidime & 0.75 & Indifferent \\
\hline & PEP27-2 / Ceftriaxone & 0.75 & Indifferent \\
\hline \multirow[t]{3}{*}{ S. aureus MW2 } & PEP27-2 / Meropemem & 0.5 & Synergy \\
\hline & PEP27-2 / Ceftazidime & 0.75 & Indifferent \\
\hline & PEP27-2 / Ceftriaxone & 0.5 & Synergy \\
\hline \multirow[t]{3}{*}{ S. aureus CCARM3089 } & PEP27-2 / Meropemem & 0.5 & Synergy \\
\hline & PEP27-2 / Ceftazidime & 0.5 & Synergy \\
\hline & PEP27-2 / Ceftriaxone & 0.5 & Synergy \\
\hline \multirow[t]{3}{*}{ S. aureus CCARM3090 } & PEP27-2 / Meropemem & 0.38 & Synergy \\
\hline & PEP27-2 / Ceftazidime & 0.5 & Synergy \\
\hline & PEP27-2 / Ceftriaxone & 0.5 & Synergy \\
\hline \multirow[t]{3}{*}{ S. aureus CCARM3518 } & PEP27-2 / Meropemem & 0.75 & Indifferent \\
\hline & PEP27-2 / Ceftazidime & 0.75 & Indifferent \\
\hline & PEP27-2 / Ceftriaxone & 0.75 & Indifferent \\
\hline
\end{tabular}

$\overline{\mathrm{An} \mathrm{FIC}}{ }^{a}$ value of $\leq 0.5$ indicated synergy, a value of $>0.5$ indicated no interaction, and a value of $>4$ indicated antagonism. 
Table S4. Combination activity of PEP27-2 with meropemem, ceftazidime and ceftriaxone against $P$. aeruginosa

\begin{tabular}{|c|c|c|c|}
\hline Organism & Agent & FIC $^{a}$ & Combination activity \\
\hline \multirow[t]{3}{*}{ P. aeruginosa ATCC15692 } & PEP27-2 / Meropemem & 0.63 & Indifferent \\
\hline & PEP27-2 / Ceftazidime & 0.75 & Indifferent \\
\hline & PEP27-2 / Ceftriaxone & 0.5 & Synergy \\
\hline \multirow[t]{3}{*}{ P. aeruginosa ATCC27853 } & PEP27-2 / Meropemem & 0.75 & Indifferent \\
\hline & PEP27-2 / Ceftazidime & 1 & Indifferent \\
\hline & PEP27-2 / Ceftriaxone & 0.75 & Indifferent \\
\hline \multirow[t]{3}{*}{ P. aeruginosa 4007} & PEP27-2 / Meropemem & 0.75 & Indifferent \\
\hline & PEP27-2 / Ceftazidime & 0.5 & Synergy \\
\hline & PEP27-2 / Ceftriaxone & 0.5 & Synergy \\
\hline \multirow[t]{3}{*}{ P. aeruginosa 4891} & PEP27-2 / Meropemem & 0.5 & Synergy \\
\hline & PEP27-2 / Ceftazidime & 0.75 & Indifferent \\
\hline & PEP27-2 / Ceftriaxone & 0.5 & Synergy \\
\hline
\end{tabular}

An FIC $^{a}$ value of $\leq 0.5$ indicated synergy, a value of $>0.5$ indicated no interaction, and a value of $4>$ antagonism. 


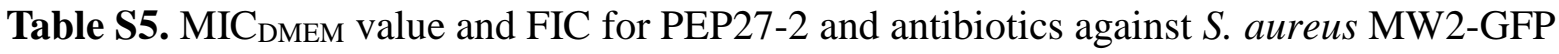

\begin{tabular}{|c|c|c|c|}
\hline & Antibiotics & $\begin{array}{l}\text { S. aureus MW2-GFP } \\
\qquad\left(1 \times 10^{9} \mathrm{CFU} / \mathrm{mL}\right)\end{array}$ & $\begin{array}{c}\text { Combination } \\
\text { activity }\end{array}$ \\
\hline \multirow[t]{4}{*}{$\operatorname{MIC}_{\text {DMEM }}(\mu \mathrm{g} / \mathrm{mL})^{a}$} & PEP27-2 & 64 & \\
\hline & Meropemen & 4 & \\
\hline & Ceftazidime & 128 & \\
\hline & Ceftriaxone & 64 & \\
\hline \multirow[t]{3}{*}{ FIC $_{\text {DMEM }}$ for PEP27- $2^{b}$} & Meropemen & 0.5 & Synergy \\
\hline & Ceftazidime & 0.75 & Indifferent \\
\hline & Ceftriaxone & 0.5 & Synergy \\
\hline
\end{tabular}

${ }^{a} \mathrm{MIC}_{\text {DMEM }}$ value was the concentration that killed $>99.9 \%$ of the bacteria in DMEM $+10 \%$ FBS after $24 \mathrm{~h}$.

${ }^{b}$ FIC DMEM for PEP27-2 values are based on 0.25 × MIC for PEP27-2. 
Table S6. List of bacterial strains used in this study

\begin{tabular}{|c|c|c|}
\hline Microorganism & Characterization & Source/Reference \\
\hline \multicolumn{3}{|l|}{ Bacterial strains } \\
\hline \multicolumn{3}{|l|}{ Starphylococcus aureus strains } \\
\hline S. aureus ATCC25923 & & $\begin{array}{l}\text { American Type Culture Collection } \\
\text { (ATCC) }\end{array}$ \\
\hline S. aureus ATCC29213 & & ATCC \\
\hline S. aureus USA300 & Community-acquired MRSA & (1) \\
\hline S. aureus MW2 (USA400) & Community-acquired MRSA & (2) \\
\hline S. aureus CCARM3089 & Multidrug resistant (MDR) strain & $\begin{array}{l}\text { Culture Collection of Antibiotic- } \\
\text { Resistant Microbes (CCARM) }\end{array}$ \\
\hline S. aureus CCARM3090 & MDR strain & CCARM \\
\hline S. aureus CCARM3518 & MDR strain & CCARM \\
\hline S. aureus RN4220 & Restriction deficient, prophage cured & (3) \\
\hline S. aureus MW2-GFP & MW2 with pYJ335-P1-GFP (4), Em ${ }^{r}$ & This study \\
\hline \multicolumn{3}{|l|}{ Pseudomonas aeruginosa strains } \\
\hline P. aeruginosa ATCC15692 & & ATCC \\
\hline P. aeruginosa ATCC27853 & & ATCC \\
\hline P. aeruginosa 4007 & MDR strain, Clinical isolate & $\begin{array}{l}\text { Chonnam National } \\
\text { UniversityHaspital (CNUH) }\end{array}$ \\
\hline P. aeruginosa 4891 & MDR strain, Clinical isolate & $\mathrm{CNUH}$ \\
\hline \multicolumn{3}{|l|}{ Escherichia coli strains } \\
\hline E. coli ATCC25922 & & ATCC \\
\hline E. coli ATCC27325 & & ATCC \\
\hline E. coli CCARM1229 & MDR strain & CCARM \\
\hline E. coli CCARM1238 & MDR strain & CCARM \\
\hline E. coli DH5 $\alpha$ & General laboratory cloning strain & (5) \\
\hline \multicolumn{3}{|l|}{ Salmonella typhimurium strains } \\
\hline S. typhimurium КСТC1926 & & КСТC \\
\hline S. typhimurium CCARM8007 & & CCARM \\
\hline S. typhimurium CCARM8008 & & CCARM \\
\hline Bacillus subtilis KCTC1998 & & KCTC \\
\hline Listeria monocytogenesis КСТС 3710 & & КСТC \\
\hline
\end{tabular}

Em, erythromycin 
Table S7. Primers used in qPCR to evaluate inflammatory cytokines in this study

\begin{tabular}{ll}
\hline Target & Primer sequence (5'-3’) \\
\hline$\beta$-actin & F: AGGCCAACCGTGAAAAGATGACCCAGAT \\
& R: GTAGACCATGTAGTTGAGGTCAATGAA \\
TNF- $\alpha$ & F: CTGCAGCACTTGGATCAGGAACCTG \\
& R: GGGAGTAGCCTGTGTGCACCTGGAA \\
IL-1 $\beta$ & F: CCTTCCAGGATGAGGACATGA \\
& R: TGAGTCACAGAGGATGGGCTC \\
IL-6 & F: ACAACCACGGCCTTCCCTACTT \\
& R: CACGATTCCCAGAGAACATGTG \\
iNOS & F: CTGCAGCACTTGGATCAGGAACCTG \\
& R: GGAGTAGCCTGTGTGCACCTGGAA \\
COX-2 & F: CAGACAACATAAACTGCGCCTT \\
&
\end{tabular}




\section{REFERENCES}

(1) Diep, B. A.; Gill, S.R.; Chang, R. F.; Phan, T. H.; Chen, J. H.; Davidson, M. G.; Lin, F.; Lin, J.; Carleton, H. A.; Mongodin, E. F.; Sensabaugh, G. F.; Perdreau-Remington, F. Complete genome sequence of USA300, an epidemic clone of community-acquired meticillinresistant Staphylococcus aureus. Lancet 2006, 367, 731-739.

(2) Baba, T.; Takeuchi, F.; Kuroda, M.; Yuzawa, H.; Aoki, K.; Oguchi, A.; Nagai, Y.; Iwama, N.; Asano, K.; Naimi, T.; Kuroda, H.; Cui, L.; Yamamoto, K.; Hiramatsu, K. Genome and virulence determinants of high virulence community-acquired MRSA. Lancet 2002, 359, 1819-1827.

(3) Kreiswirth, B.N.; Lofdahl, S.; Betley. M. J.; O'Reilly, M.; Schlievert, P. M.; Bergdoll, M. S.; Novick, R. P. The toxic shock syndrome exotoxin structural gene is not detectably transmitted by a prophage. Nature 1983, 305, 709-712.

(4) Yeo, W. S.; Arya, R.; Kim, K. K.; Jeong, H.; Cho, K. H.; Bae, T. The FDA-approved anticancer drugs, streptozotocin and floxuridine, reduce the virulence of Staphylococcus aureus. Sci. Rep. 2018, 8, 2521.

(5) Taylor, R. G.; Walker, D. C.; McInnes, R. R. E. coli host strains significantly affect the quality of small scale plasmid DNA preparations used for sequencing. Nucleic Acids Res. 1993, 21, 1677-1678. 